\title{
Total Anomalous Pulmonary Venous Connection (TAPVC) (Supra cardiac type)- A Case Study
}

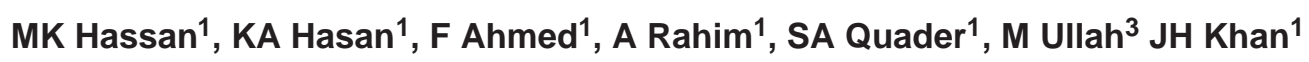 \\ A Salam², KN Mahmood ${ }^{1}$ \\ ${ }^{1}$ Department of Cardiovascular Surgery, NICVD, ${ }^{2}$ Department of Paediatric Cardiology, NICVD, \\ 3Department of Cardiology, NICVD, Dhaka
}

\begin{abstract}
s:
Keywords:

Total anomalous

pulmonary

Repair of Total Pulmonary venous connection (TAPVC) continues to be associated to significant mortality and morbidity. We here reported a female patient of two and half years, underwent rechanneling of supracardiac TAPVC, diagnosis was suggested by Echocardiography and confirmed venous connection by catheter-angiography which allowed definition of the anatomy. The patient has got fluent (TAPVC), pulmonary venous drainage and her heart function resumed to NYHA I. TAPVC should be operated

Pulmonary artery

hypertension. on immediately at definite diagnosis, the fluency of common pulmonary vein-left atrium anastomosis and proper post operative care can ensure a satisfactory outcome.
\end{abstract}

(Cardiovasc. j. 2010; 3(1): 104-106)

\section{Introduction:}

TAPVC is characterized by the failed union of the pulmonary veins with the developing left atrium in combination with a persistent embryologic connection between the pulmonary and systemic venous system. ${ }^{1}$

The impact of the pulmonary circulation depends on the degree to which the pulmonary venous derainage is obstructed and the magnitude of the left to right shunt. ${ }^{1}$

TAPVC is a rare congenital anomaly, corresponding to approaximately $2 \%$ of all congenital heart defects. A recognized classification divides TAPVC into four groups according to the site of connection. With type I, having a supracardiac connection (50\%), the common pulmonary trunk joins the left vertical vein, the innominate vein or the superior vena cava. With type II, having a cardiac connection (30\%), the anomalous pulmonary venous drainage enters the coronary sinus and flows directly into the right atrium. With type III, the site being infracardiac (15\%), connection occurs to the portal vein, venous duct, or inferior vena cava below the diaphragm. With type IV, a mixed site variety (5\%), the anomalous venous return occurs at several levels. Pathophysiologically, these four types are subclassified according to whether the pulmonary venous return is obstructed or nonobstructed. ${ }^{2}$

\section{Case Report:}

A two and half years aged female baby was admitted to our department because of respiratory distress and cyanosis and severe pulmonary artery hypertension. She weighs $12 \mathrm{Kg}$ and her general condition was poor. A systolic murmur was noted. Chest radiography showed pulmonary venous congestion with interstitial and alveolar edema. Echocardiography revealed that the right atrium, right ventricle and left atrium could not be identified, coronary sinus was found to be narrow in size. There was $15 \mathrm{~mm}$ defect in interatrial septum and right to left shunt was seen through the defect. There was supracardiac TAPVC and there was a PDA with left to right shunt. Pulmonary artery systolic pressure was observed 95mm Hg.

Catheter angiographic examination was undertaken and the diagnosis was confirmed, it was supracardiac TAPVC with ASD secundum with obstruction at the level of vertical vein in between the LPA and left principal bronchus with severe PAH. But in case of pulmonary hypertension due to pulmonary venous obstruction it carries an increased risk. Surgical technique: Standard cardiopulmonary bypass with aorto bicaval cannulation with profound hypothermia was instituted. Phenoxybenzamine

Address of Correspondence : Dr. Muhammod Kamrul Hassan, Department of Cardiac Surgery, NICVD, Dhaka, Bangladesh. 
was administered electively due to pulmonary hypertension and obstruction. In this case, repair was accomplished by direct common veno-left atrial anastomosis (Shumaker technique). Heart was de aired and cross-clamp released. Patient was re warmed just before coming off bypass, the vertical vein was snagged. After achieving stable hemodynamic status with inotropic support controlled pulmonary artery pressure and satisfactory blood gas values atria sternum was closed. Of late, Sildenafil was used as alternative to nitric oxide.

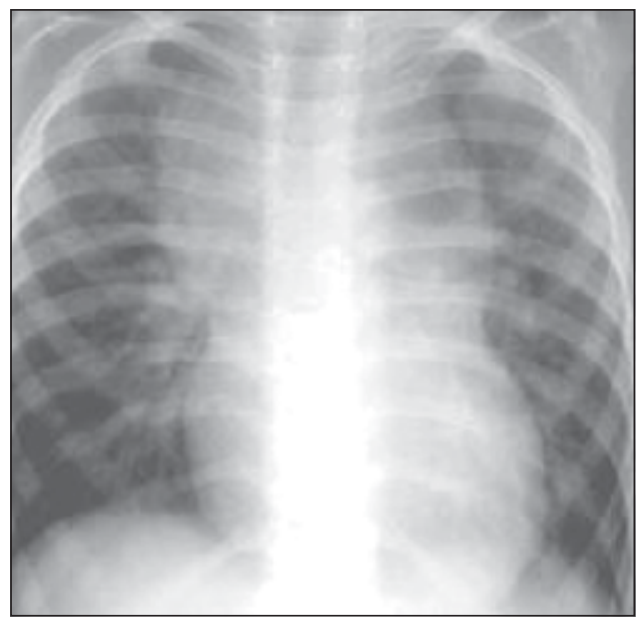

Fig.-1: Chest X-ray showing features consistent with TAPVC.
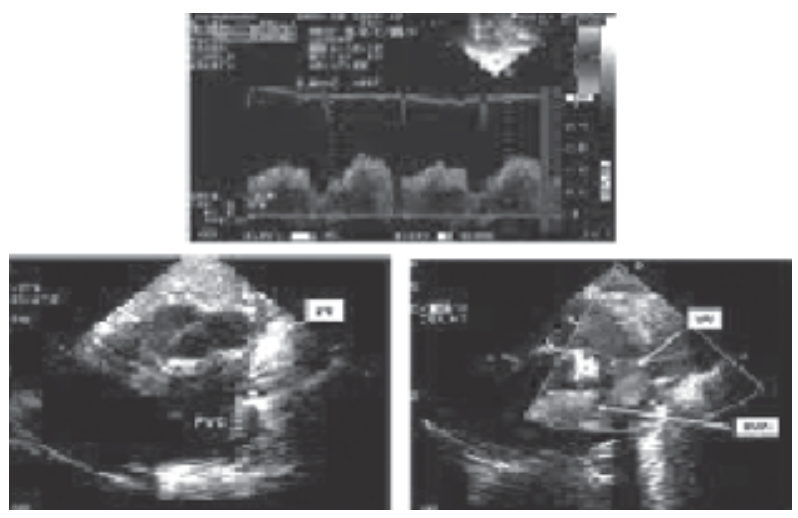

Fig.-2: Echocardiography showing features consistent with TAPVC

Postoperatively patient was ventilated for a period hours. At the time of last follow up pulmonary hypertension had resolved. No residual ASD or PDA was observed. Patient has got fluent pulmonary venous drainage.

\section{Discussion:}

The first series of operations performed for TAPVC in 1960s reported an extremely high mortality ranging from 65 to $88 \%{ }^{3}$ Results of correction of TAPVC in infancy have improved markedly in recent years, with an operative mortality of $5 \%$ or less by same institution. ${ }^{3}$

Operative and late mortality rates were $19.3 \%$ and 4.3\% among 57 consecutive patients aged 15 days to 18 years (median 6 months), underwent rechanneling of mixed totally anomalous pulmonary venous connection in All India Institute of Medical Sciences, India. ${ }^{4}$

Alteration of preoperative stabilization, early operation, progressive refinement of surgical technique and improved quality of postoperative management may have all contributed to this decline. $^{3}$

The diagnosis of TAPVC is made when all form of pulmonary veins drain anomalously to the right atrium or to a tributary of the systemic veins. It consists between $1 \%$ and $1.5 \%$ of all children with congenital heart disease and can be categorized by the site of drainage into the systemic circulation (supracardic $45 \%$, infracardiac $25 \%$, cardiac $25 \%$, mixed 5\%). The clinical presentation is different if the pulmonary venous drainage is unobstructed (heart failure, mild cyanosis) or obstructed (respiratiory failure, severe heart failure). Surgical management depends on the anatomic type. Obstructed TAPVC require urgent surgical intervention, where as unobstructed TAPVC can be dealt with electively; although this is usually operated on once the diagnosis is made. Postoperative pulmonary artery hypertension can be problematic. Recent surgical results with isolated TAPVC have improved, with operative mortality consistently at less than $10 \%$. A particularly challenging group of patients are those with single ventricle physiology and TAPVC with high operative mortality and poor long term survival. ${ }^{5}$

Supracardiac type were obstructed either at the junction of the common venous chamber and vertical vein or at the junction of the vertical vein with the innominate vein or the superior vena cava or at the interatrial communication. ${ }^{3}$ Our patients 
has got obstruction at the junction of the vertical vein with innominate vein.

Survival depends on the presence of a right to left intracardiac shunt, which almost always occurs through a patent foramen ovale that is rarely restrictive. $^{2}$

The most serious complication after TAPVC repair is pulmonary vein stenosis. This can be intrinsic to the pulmonary vein or at the anastomosis and is reported to occur in $8 \%$ to $54 \%$ of cases. ${ }^{1}$

Early mortality after neonatal palliation is heterotaxy syndrome with TAPVC was $26 \%$ in a 10 year series at Boston children hospital and substantially higher in other reports. ${ }^{6}$

\section{Conclusion:}

The result of the surgical correction of this anomaly is associated with acceptable morbidity and mortality depending on early referral and surgery without progression of the pulmonary vascular pathology.

\section{References:}

1. Camille L, Hamlock H, David Z, Ravi R, Joseph FM, Pedro M. Total anomalous pulmonary venous connection : an analysis of current management strategies in a single institution Ann Thorac Surg 2005; 79: 596-606.

2. Erven S, Ozler M, Boston EC. Echocardiographic diagnosis of total anomalous pulmonary venous connection of the infracardiac type. Anadolu Kardiyol 2007; 7: 82-84.

3. Girish W, Dhora BS, Koshy S, Kumar S, Krishnanaik S, Rao SG. Repair of total anomalous pulmonary venous connection in neonates. Ind $J$ Thorac Cardiovasc surg 2004; 20: 155-58.

4. Chowdhury UK, Airan B, Malholm A, Bisoi AK, Sayema A, Kotheri SS, Kalaivanj M, Venogopal P. Mixed total anomalous pulmonary venous connection: anatomic variations, surgical approach, techniques and results. $J$ Thorac Cardiovasc Surg 2008; 135(1): 106-16.

5. Kanter KR, Surgical repair of total anomalous pulmonary venous connection. Senin Thorac Cardiovasc Surg 2006; 40:4.

6. Sachclev SM, Jena KP, Kurup P, Varghese R, Kumar $\mathrm{S}$, Coelho R, "Outcome of single ventricle and total anomalous pulmonary venous connection. Asian Cardiovasc Thorac Ann. 2006, 14, 367-70. 